\title{
Convective Pumping of Magnetic Fields: On the Flux Storage Problem for Solar-like Dynamos
}

\author{
S. B. F. Dorch
}

The Royal Swedish Academy of Sciences, Stockholm Observatory, SE-13336 Saltsjöbaden, Sweden

\author{
A. Nordlund \\ The Niels Bohr Institute for Astronomy, Physics and Geophysics, \\ Juliane Maries Vej 30, DK-2100 Copenhagen Ø, Denmark
}

\begin{abstract}
We present results from three-dimensional numerical simulations of the interaction of stratified over-turning solar-like convection with a large-scale magnetic field: By the very nature of stellar convection, even a strong magnetic field may be held down at the bottom of the convection zone, rendering the flux storage problem obsolete. This effect may also explain the observations of some magnetically active but fully convective late type dwarf stars.
\end{abstract}

\section{Introduction}

Active late type $\mathrm{G}, \mathrm{K}$ and $\mathrm{M}$ dwarf stars exhibit spectra formed under the influence of magnetic fields. The most well-studied example of such a star is the Sun: Based on solar observations it is generally concluded that the magnetic surface activity is part of a stellar dynamo process and that bipolar active regions are manifestations of buoyant strands of a large scale toroidal magnetic field.

A long standing issue in the theory of stellar dynamos is the so-called "flux storage problem", i.e. the problem of explaining how the magnetic field may be kept within a stellar convection zone (CZ) long enough for the dynamo to operate: Because of the on the average super-adiabatic stratification of a solarlike CZ, a buoyant magnetic flux rope will typically escape the CZ in a month or so, while the dynamo is thought to operate on a time scale on the order of a decade (as observed both in the case of the Sun and several other magnetically active stars, e.g. Schrijver \& Zwaan 2000).

In the following we address this problem, by discussing results from numerical three-dimensional computer simulations of the interaction of over-turning stratified convection and magnetic fields of varying strength (see also Dorch 1998; Dorch \& Nordlund 2000).

\section{Model}

We solve the compressible and resistive three-dimensional magnetohydrodynamical equations in a Cartesian box using the staggered mesh method developed 

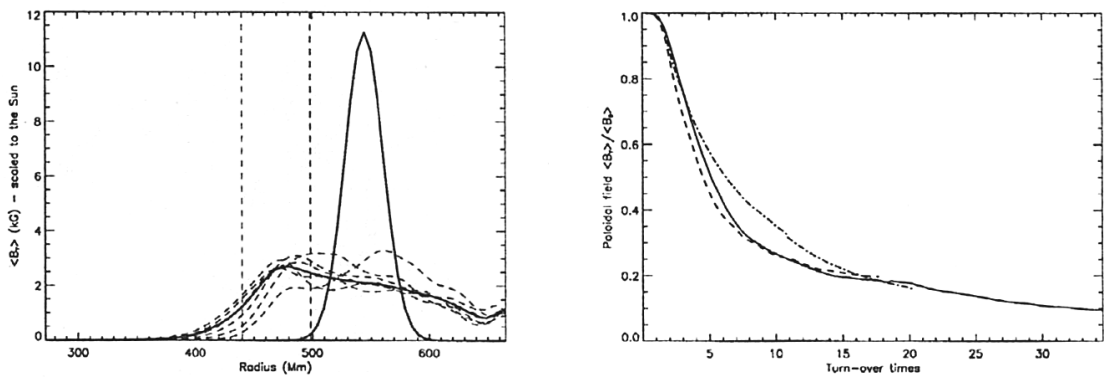

Figure 1. Left: Horizontal average of magnetic field at different instants (dashed curves) and the resulting time-average (solid curve). Gaussian solid curve is initial distribution. Right: Average field (which measures flux) as function of time in three experiments with an open upper boundary, but different initial magnetic field strength.

by Galsgaard and others (Galsgaard \& Nordlund 1997; Nordlund, Galsgaard, \& Stein 1994).

The initial condition consists of a uniform magnetic sheet placed in the middle of a convective layer (with a relaxed state of stratified over-turning convection) between two stable layers. A thin cooling layer is placed at the top. The computational box is horizontally periodic while we have experimented with both open and closed upper boundary conditions. In a few cases solar-like differential rotation is included.

\section{Results}

The magnetic field is "pumped" downwards and settles on a characteristic average flux distribution peaking just below the CZ (see Figure 1 - left): Of all the fluid parcels initially threaded by magnetic field lines about half are ascending. Because of the stratification most of the fluid over-turns and descends down to the bottom of the CZ dragging the field along.

In the numerical experiments with an open upper boundary the flux-loss is strongly exaggerated in comparison to the Sun (Figure 1 - right): The model upper boundary (the surface) is far below the true solar surface, and in the Sun most of the ascending flux over-turns leaving only a tiny fraction to reach the surface. Moving the model boundary upwards 0.6 density scale heights reduces the flux-loss by $20 \%$. However, the distribution of flux in the bulk of the CZ is robust towards changes in the boundary condition.

To study the influence of rotation on the pumping effect, we used a solarlike differential rotation with a steep radial gradient at the bottom of the CZ, and a smooth polar variation inside the CZ: A rendering of magnetic field lines (Figure 2 - right) shows how the magnetic field organizes itself into toroidal flux streets in the CZ (four in this case because of the horizontal periodicity of the imposed differential rotation). Below the bottom of the $\mathrm{CZ}$ the field is weak and retains the initial poloidal orientation. As in the non-rotating cases the stratified 

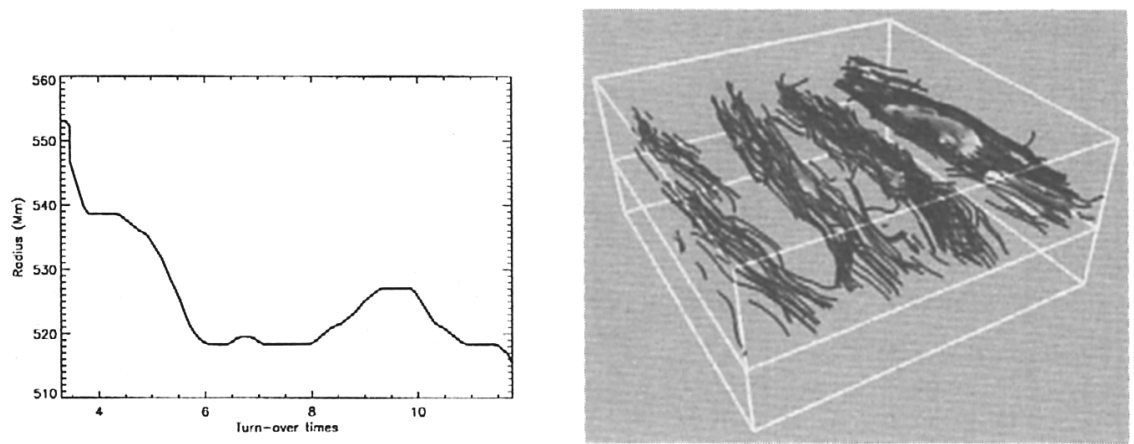

Figure 2. Left: Vertical position of the maximum of the horizontally averaged magnetic field in the case including rotation. Right: Snapshot of strong magnetic field lines.

convection is able to hold down even a formally super-equipartition field: Figure 2 (left) shows the depth of the location of the maximum of the horizontally averaged flux. Note for comparison that the undershoot layer begins at around a depth of $500 \mathrm{Mm}$ in the model, i.e. the flux peaks inside the CZ.

\section{Discussion and Conclusions}

Stratified over-turning convection has a tendency to transport magnetic flux down towards the bottom of the CZ. This "pumping mechanism" is robust towards different types of boundary conditions, rotation and strong magnetic fields. In simulations (Dorch 1998; Tobias et al. 1998 and ibid.) a lot of the flux is pumped into the stable undershoot layer, but in a real star where this layer is presumably much thinner than in the numerical models, the bulk of the flux resides within in the CZ. This renders the flux storage problem obsolete.

Furthermore, the pumping mechanism may also help to explain the existence of magnetically active, but fully convective $M$ dwarf stars that are not believed to posses stable undershoot layers.

Acknowledgments. SBFD acknowledges support through an EC-TMR grant to the European Solar Magnetometry Network.

\section{References}

Dorch, S.B.F. 1998, Ph.D. Thesis, Copenhagen University, 59

Dorch, S.B.F., \& Nordlund, A. 2000, A\&A, in press

Galsgaard, K., \& Nordlund, Å. 1997, Journ. Geoph. Res., 102, 219

Nordlund, A., Galsgaard, K., \& Stein, R.F. 1994, in NATO ASI Series 433, Solar Surface Magnetic Fields, ed. R.J. Rutten, 471

Schrijver, C.J., \& Zwaan, C. 2000, Solar and Stellar Magnetic Activity, Cambridge, 299

Tobias, S., Brummel, N., Clune, T., \& Toomre, J. 1998, ApJ, 502, L177 